\title{
Energy scalable, offset-free ultrafast mid-infrared source harnessing self-phase-modulation enabled spectral selection
}

\author{
GengJ Zhou, ${ }^{1,2}$ QIAN CAO, ${ }^{1,2}$ Franz X. KÄRTNER, ${ }^{1,2,3}$ AND GUOQING \\ CHANG ${ }^{1,3,4, *}$ \\ ${ }^{1}$ Center for Free-Electron Laser Science, DESY, Notkestraße 85, 22607 Hamburg, Germany \\ ${ }^{2}$ Physics Department, University of Hamburg, Luruper Chaussee 149, 22761 Hamburg, Germany \\ ${ }^{3}$ The Hamburg Centre for Ultrafast Imaging, Luruper Chaussee 149, 22761 Hamburg, Germany \\ ${ }^{4}$ Current affiliation: Beijing National Laboratory for Condensed Matter Physics, Institute of Physics, Chinese Academy of Sciences, Beijing \\ 100190, China \\ *Corresponding author: guoqing.chang@iphy.ac.cn
}

Received XX Month XXXX; revised XX Month, XXXX; accepted XX Month XXXX; posted XX Month XXXX (Doc. ID XXXXX); published XX Month XXXX

\begin{abstract}
We demonstrate a high-power offset-free ultrafast midIR laser source based on difference frequency generation (DFG). Powerful signal pulses are obtained by filtering the rightmost spectral lobe of optical spectra broadened by fiber-optic nonlinearities dominated by self-phase modulation. The resulting mid-IR pulses are tunable from $7 \mu \mathrm{m}$ to $18 \mu \mathrm{m}$ with up to 5.4-mW average power. We experimentally and numerically investigate power scaling of this DFG source and demonstrate that increasing the signal power is an efficient approach for generating high power mid-IR pulses. (C) 2018 Optical Society of America
\end{abstract}

OCIS codes: (190.4370) Nonlinear optics, fibers; (320.2250) Femtosecond phenomena.

http://dx.doi.org/10.1364/OL.99.099999

High repetition-rate $(>10 \mathrm{MHz})$ ultrafast laser sources that are tunable in the mid-infrared (IR) wavelength range of 6-20 $\mu \mathrm{m}$ (known as the molecular 'fingerprint' region) hold great promise for many important spectroscopy applications [1,2]. To date, ultrafast mid-IR laser sources with a wavelength beyond $7 \mu \mathrm{m}$ are usually implemented via difference-frequency generation (DFG) between two synchronized pulse trains with different central wavelength. The major component of a DFG-based mid-IR source is a high power femtosecond laser. Due to their superior power scalability and the potential of an all-fiber format, ultrafast Yb-fiber lasers have become popular laser sources to implement DFG-based ultrafast mid-IR sources [3-8]. In a typical configuration, the output pulse trains from an Yb-fiber laser are split into two portions with one portion serving as the pump, and the other portion being used to generate signal pulses with a broadly tunable center wavelength. A well-established technique to produce tunable signal pulses is to use fiber-optic nonlinearities to broaden the optical spectrum of an ultrafast Yb-fiber laser; the newly generated spectral component at the longer wavelength side serves as the signal for DFG [3-6]. Fiber-optic nonlinearities arise from the third-order susceptibility of fiber glass and preserve the carrier-envelope offset $f_{C E}$ during the nonlinear spectral broadening. Consequently the signal pulse and the pump pulse share the same $f_{C E}$, which cancels out during DFG resulting in an offset-free mid-IR source. If the source laser's repetition rate is stabilized, the offset-free mid-IR source becomes a mid-IR frequency comb.

Many groups employed Raman soliton self-frequency shift in an optical fiber to generate signal pulses [6-12]. The center wavelength of the resulting Raman soliton sources can be continuously red-shifted by increasing the coupled pulse energy into the fiber. To make full use of the excellent power scalability of the Yb-fiber laser technology, specialty fibers with negative group-velocity dispersion at $\sim 1.03 \mu \mathrm{m}$ are fabricated to accommodate Raman solitons. Normally these specialty fibers exhibit nonlinearity about 10 times higher than standard single-mode fibers, limiting the Raman soliton pulse energy to < $1 \mathrm{~nJ}$; in the wavelength range of $1070-1200 \mathrm{~nm}$, the pulse energy is typically less than $0.2 \mathrm{~nJ}$. Use of these low-energy Raman solitons as the signal pulses in DFG inevitably leads to low-energy (and low average power) mid-IR pulses, which severely limits their practical spectroscopic applications.

One might expect that the average power of a DFG mid-IR source can be efficiently increased if a more powerful fiber laser is used to provide pump pulses. Theoretically using $\mu \mathrm{J}$-level pulses as the pump for DFG may generate $\sim 100-\mathrm{mW}$ mid-IR pulses at $\sim 10 \mu \mathrm{m}$. However, the properties of nonlinear crystals impose other limitations. To date, $\mathrm{AgGaSe}_{2}$ (AGSe) and GaSe are the mostly used nonlinear crystals that can generate DFG-based ultrafast mid-IR pulses covering the entire fingerprint region of 6.6-20 $\mu \mathrm{m}[13,14]$. Both AGSe and GaSe have low damage threshold, which limits the allowed pump-pulse energy in DFG. Our experimental results show that even for a loosely focused beam with $\sim 200-\mu \mathrm{m}$ spot diameter, 30-MHz, 200-fs pulses at $1.03 \mu \mathrm{m}$ with 200-nJ pulses energy can damage a GaSe crystal. Because the pump pulse energy has to be kept below a certain value to prevent crystal damage, increasing the signal pulse energy becomes a powerful and practical solution to achieve high-power mid-IR pulses. Since Raman soliton sources exhibit low pulse energy, novel energetic fiberoptic sources are highly desired. Such an ultrafast source should emit nearly transform-limited femtosecond pulses featuring broadly tuning range, low noise, and superior energy scalability. 
Recently we demonstrated a new fiber-optic method to derive tunable femtosecond pulses. This method employs self-phase modulation (SPM) dominated nonlinearities to broaden a narrow input optical spectrum to generate well-isolated spectral lobes; spectrally filtering the leftmost/rightmost spectral lobes produces nearly transform-limited femtosecond pulses $[15,16]$. Based on an $\mathrm{Yb}$ fiber laser system, this SPM-enabled spectral selection (SESS) approach can generate $\sim 100$-fs pulses with up to 20 -nJ pulse energy tunable in the range of $1030-1215 \mathrm{~nm}$ [16]. In this submission, we use SESS to generate energetic signal pulses and demonstrate a DFG-based mid-IR source with excellent energy scalability.

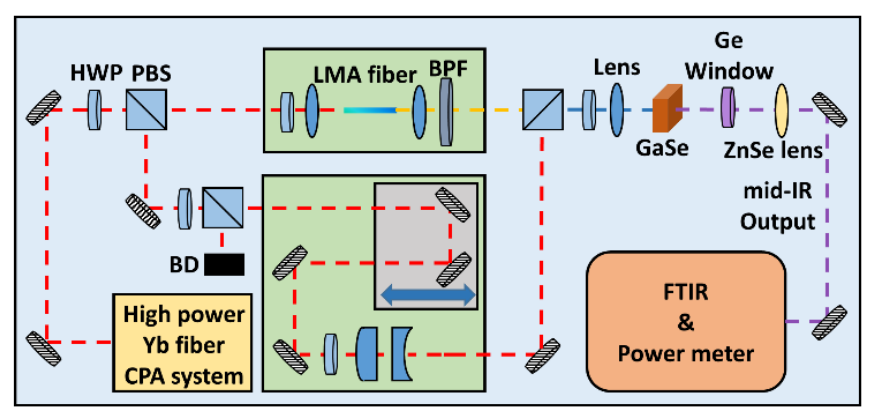

Fig. 1. Schematic of high-power, widely tunable DFG-based mid-IR source. WDM: wavelength division multiplexer, HWP: half-wave plate, PBS: polarization beam splitter, BD: beam dumper, LMA: large-mode-area, BPF: bandpass filter.

Figure 1 illustrates the experimental setup. The home-built high power $\mathrm{Yb}$-fiber system delivers 165 -fs pulses with 14.5-W average power at $30-\mathrm{MHz}$ repetition rate, corresponding to $480-\mathrm{nJ}$ pulse energy. The laser output is split into two replicas by a half-wave plate (HWP) together with a polarization beam splitter (PBS). One replica is coupled into a piece of large-mode-area (LMA) fiber for SPM-enabled spectra broadening, followed by a band-pass filter to generate tunable femtosecond signal pulses. The other replica serves as the pump for subsequent DFG in a GaSe crystal. A HWP together with a PBS continuously adjusts the optical power employed in DFG. The pump pulses and the signal pulses are collinearly combined by a broadband PBS, and an optical delay line ensures their temporal overlap. An achromatic HWP is employed to adjust the polarization of both signal and pump pulses for maximizing $d_{\text {eff }}$ in the DFG process. The combined pulses are focused into the GaSe crystal with $\sim 200-\mu \mathrm{m}$ spot size in diameter. The generated mid-IR pulses pass through a 5-mm thick Germanium window that blocks the residual pump pulses and signal pulses, then being collimated by a ZnSe lens. A calibrated thermopile detector measures the average power of the mid-IR pulses, and their optical spectrum is characterized by a Fourier transform (FT) IR spectrometer.

To obtain energetic signal pulses, we employ 4-cm LMA fiber with a mode-field diameter of $12.5 \mu \mathrm{m}$. Due to a large input pulse energy $(>100 \mathrm{~nJ})$ and short fiber length, SPM dominates the spectral broadening and generates multiple distinct spectral lobes. As we increase the coupled power from $3.2 \mathrm{~W}$ to $6.8 \mathrm{~W}$, the rightmost spectral lobe (i.e., the farthest spectral lobe on the longer wavelength side) redshifts from $1.1 \mu \mathrm{m}$ to $1.2 \mu \mathrm{m}$. Filtering these rightmost spectral lobes produces widely tunable signal pulses For example, the red curve in Fig. 2(a) plots the output spectrum at 3.2-W coupled power. The spectrum has the rightmost spectral lobe at $1.1 \mu \mathrm{m}$, which is selected by a bandpass filter [blue curve in Fig. 2(a)]. Inset of Fig. 2(a) shows the measured autocorrelation trace (blue curve) and the calculated autocorrelation trace (black curve) of the transform-limited pulses corresponding to the filtered spectrum. The pulse duration is estimated to be $125 \mathrm{fs}$, assuming a deconvolution factor of 1.4. As we increase the power to $6.8 \mathrm{~W}$, the resulting broadened spectrum has the rightmost one at $1.2 \mu \mathrm{m}$ [red curve in Fig. 2(b)]. The filtered spectral lobe [blue curve in Fig. 2(b)] results in pulses with an estimated duration of $110 \mathrm{fs}$ [inset of Fig. 2(b)].
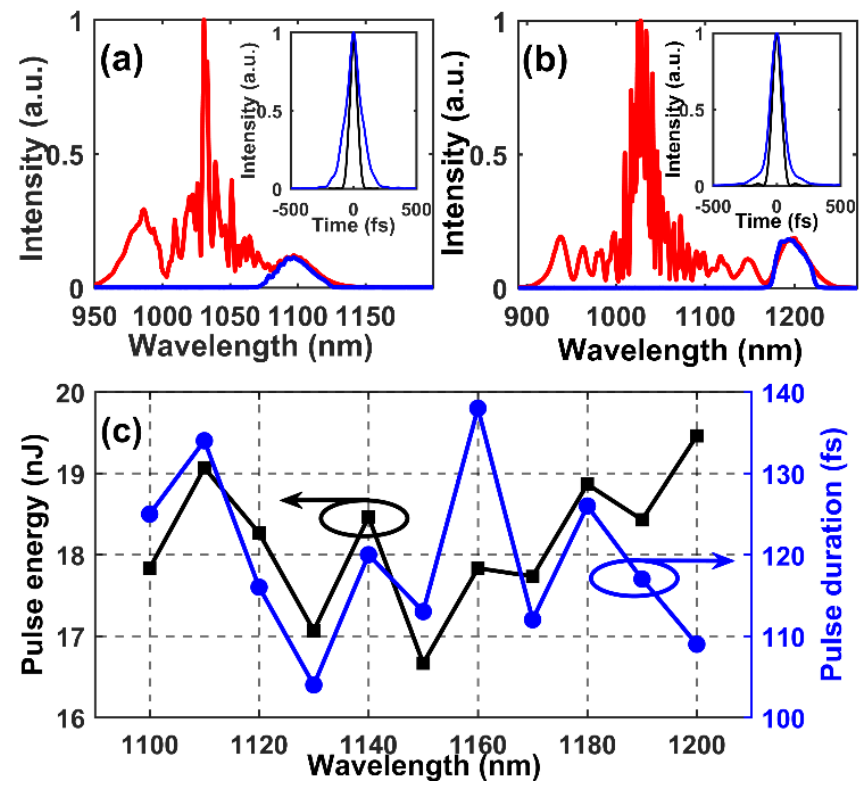

Fig. 2. SPM-dominated spectral broadening in the 4-cm LMA fiber with the rightmost spectral lobe tunable by varying the coupled power. (a) Output spectrum at 3.2-W coupled power (red curve) and the filtered spectral lobe (blue curve). Inset shows measured autocorrelation trace (blue) and calculated autocorrelation trace corresponding to the transform-limited pulse given by the filtered spectrum (black). (b) similar as (a) for the coupled power increased to $6.8 \mathrm{~W}$. (c) Measured pulse energy (black curve) and duration (blue curve) for the pulses resulting from the filtered spectral lobes at $1100 \mathrm{~nm}, 1110 \mathrm{~nm} . .1200 \mathrm{~nm}$. Black curve: measured pulse energy; blue curve: estimated pulse duration.

To show the capability for generating wavelength tunable signal pulses, we adjust the coupled power into the LMA fiber and then use a series of bandpass filters to select the rightmost spectral lobes between 1100-1200 nm with a wavelength step of $10 \mathrm{~nm}$. Figure 2(c) shows the measured pulse energy and duration for these 11 representatively selected spectral lobes. The pulse duration varies between 100-140 fs [blue curve in Fig. 2(c)]. The black curve shows that the pulse energy is 16-20 nJ, which represents >10 times energy improvement in comparison with a PCF-based Raman soliton source at the same wavelength range. These energetic signal pulses are combined with the pump pulses to perform Type-I phase matched $(\mathrm{e}-\mathrm{O}=0) \mathrm{DFG}$ in a GaSe crystal to generate mid-IR pulses. Two GaSe crystals with different thickness- $0.5 \mathrm{~mm}$ and $2 \mathrm{~mm}$-are employed. During the experiment, we adjust the splitting ratio of the laser power such that SESS produces the signal wavelength tunable between $1100-1200 \mathrm{~nm}$ with 10-nm wavelength steps, and use the rest of the laser power serving as the pump. To avoid crystal damage, we use another combination of HWP and PBS to keep the pump power below $6 \mathrm{~W}$, corresponding to a maximum pulse energy of $200 \mathrm{~nJ}$.

Figure 3(a) plots the measured mid-IR spectra generated from the $0.5-\mathrm{mm}$ thick GaSe. The center wavelength varies from $7.4 \mu \mathrm{m}$ to 16.2 $\mu \mathrm{m}$ and the spectral bandwidth (full width at half maximum, FWHM) is about 0.4-2.7 $\mu \mathrm{m}$. The blue dashed line records the average power for each mid-IR spectrum. The maximum measured power is $1.9 \mathrm{~mW}$ for the mid-IR pulses at $11.3 \mu \mathrm{m}$ as a result of DFG between the pump pulses at $1.03 \mu \mathrm{m}$ and the signal pulses at $1.14 \mu \mathrm{m}$. The fine structure in the mid-IR spectrum at about 7.4 $\mu \mathrm{m}$ [red curve in Fig. 3(a)] is caused by water absorption in the environmental air. $\mathrm{CO}_{2}$ in the atmosphere exhibits strong absorption at $15 \mu \mathrm{m}$, which generates a deep spectral valley in the mid-IR spectra that cover this wavelength. To scale up the mid-IR power, we replace the $0.5-\mathrm{mm}$ thick GaSe by a 2 -mm thick one and redo the experiments. The measured spectra and their average power are recorded in Fig. 3(b). Since a thicker crystal permits smaller phase-matching bandwidth, the mid-IR spectra generated by $2-\mathrm{mm}$ thick GaSe possess a narrower spectral bandwidth of 0.3-2 $\mu \mathrm{m}$. At the 
expense of phase-matching bandwidth, a thicker GaSe crystal increases the power of mid-IR pulse yield by a factor of 2-4. The maximum average power is $5.4 \mathrm{~mW}$ for the mid-IR pulses at $9.5 \mu \mathrm{m}$ and the minimum is $1.7 \mathrm{~mW}$ at $16.7 \mu \mathrm{m}$.
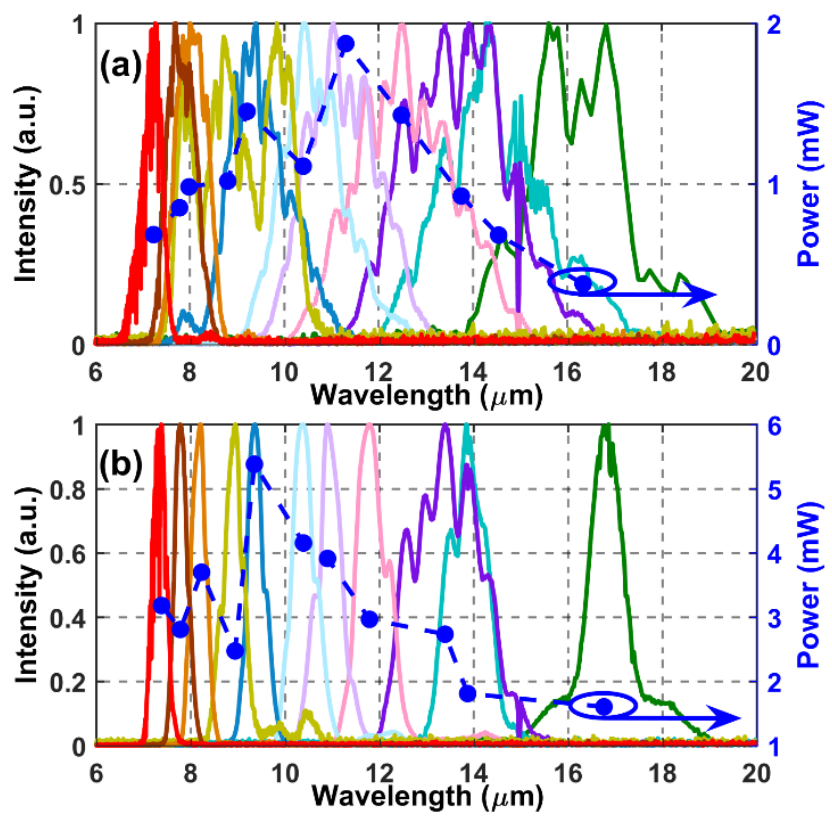

Fig. 3. Measured spectra and average power for mid-IR pulses generated in GaSe crystals of different thickness: (a) $0.5 \mathrm{~mm}$ and (b) $2 \mathrm{~mm}$. During DFG, the pump pulses are fixed at $1030 \mathrm{~nm}$ and the signal pulses are tuned in $1100-1200 \mathrm{~nm}$ with 10-nm wavelength step.

Since increasing the pump power or the signal power can both scale up the resulting mid-IR power, a question naturally arises: which approach works more efficiently? To answer this question, we first fix the signal wavelength at $1150 \mathrm{~nm}$ and place a metallic neutral density step-variable filter in the signal arm to adjust the launched signal power onto the 2-mm thick GaSe crystal. Figure 4(a) shows the mid-IR power as a function of pump power for a signal power of $0.1 \mathrm{~W}$ (blue curve), $0.2 \mathrm{~W}$ (green curve), and $0.4 \mathrm{~W}$ (red curve), respectively. For a fixed signal power, the mid-IR power increases linearly with the increased pump power until saturation occurs as the pump power reaches about $3 \mathrm{~W}$. This saturation is caused by the onset of twophoton absorption in GaSe. The same mid-IR power can be achieved using different pump-signal power combinations. For example, the pump-signal power combinations of (0.9-W pump, 0.4-W signal), (1.8W pump, 0.2-W signal), and (3.6-W pump, 0.1-W signal) all result $\sim 0.9$ $\mathrm{mW}$ mid-IR power, which immediately suggests that increasing the signal power constitutes an efficient approach for power scaling of the mid-IR pulses. This is of particular importance given that the pump power is not limited by the Yb-fiber laser system. It is the GaSe's twophoton absorption and damage threshold that limits the maximum pump fluence on the GaSe crystal. In this scenario, increasing the signal power constitutes an efficient approach to further improve the mid-IR power. Figure 4(b) shows the mid-IR power as a function of signal power for the pump pulses at different power levels of $0.9 \mathrm{~W}$ (blue curve), $1.5 \mathrm{~W}$ (green curve), and $3 \mathrm{~W}$ (red curve). For a fixed pump power, the mid-IR power also increases linearly with the increased signal power, suggesting that increasing signal power can scale up the mid-IR power as efficiently as increasing pump power. The linear dependence of the mid-IR power on both signal power and pump power- which is valid for the pump power below $3 \mathrm{~W}$-implies that the mid-IR power indeed scales up with the power product between the pump and the signal. To better illustrate this point, we redraw the experimental data in terms of mid-IR power versus the pump-signal power product. The red squares in Fig. 5(a) represent these data points. We carry out similar experimental measurements using 0.5 - mm thick GaSe and plot the data as the blue triangles in Fig. 5(a). The red and blue lines are the linear fit of the data, demonstrating the linear dependence of the mid-IR power on the pump-signal power product.
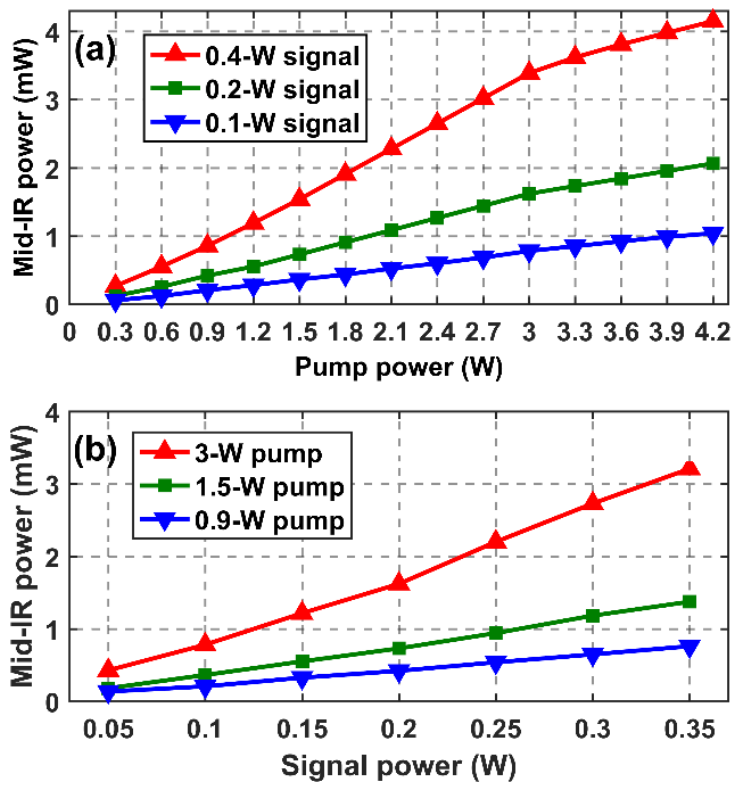

Fig. 4. (a) Mid-IR power scaling versus pump power for signal power at $0.1 \mathrm{~W}$ (blue curve), $0.2 \mathrm{~W}$ (green curve), and 0.4 W (red curve). (b) Mid-IR power scaling versus signal power for pump power at $0.9 \mathrm{~W}$ (blue curve), $1.5 \mathrm{~W}$ (green curve), and $3 \mathrm{~W}$ (red curve). A 2-mm thick GaSe crystal is used for DFG.

To confirm the experimental results in Fig. 5(a), we carry out numerical simulation by modeling the DFG process using coupledwave equations [17]. In the simulation, pump pulses are centered at $1030 \mathrm{~nm}$ with 170 -fs duration and signal pulses at $1150 \mathrm{~nm}$ with $130-$ fs duration. Both pump beam and signal beam have the same spot size of $200 \mu \mathrm{m}$ inside the crystal. Their pulse energy is chosen to match the experimental parameters from Fig. 4. The simulation also takes into account crystal dispersion, Fresnel loss for the input and output, and additional 20\% mid-IR loss from optical components.

Figure 5(b) shows the simulation results. For both $0.5 \mathrm{~mm}$ and 2 $\mathrm{mm}$ GaSe crystals, the mid-IR average power scales linearly with respect to the pump-signal power product. We also notice a discrepancy of about $30 \%$ between experiment and simulation. This may be due to the absence of multiple-photon absorption, beam walkoff effect in the simulation, and the difference between simulation spectra and experimental ones. Nevertheless, the reasonable agreement between experimental data and simulation results confirms that increasing the signal power for mid-IR power scaling is as efficient as increasing the pump power. 

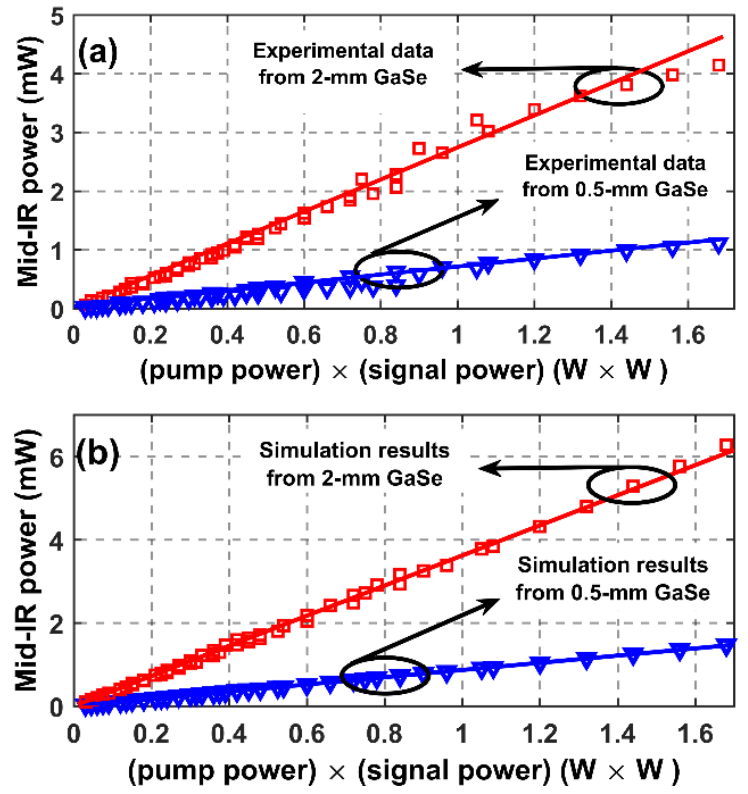

Fig. 5. Power scaling of mid-IR versus power product between pump pulses and signal pulses for different GaSe thicknesses: $0.5 \mathrm{~mm}$ and $2 \mathrm{~mm}$. (a) experimental results and (b) simulation results.

In conclusion, we demonstrate an Yb-fiber laser based ultrafast midIR laser source tunable in 7-18 $\mu \mathrm{m}$. The powerful signal pulses are obtained by filtering the rightmost spectral lobe of optical spectra broadened by SPM-dominated nonlinearity inside an optical fiber. Up to $5.4-\mathrm{mW}$ mid-IR pulses centered at $9.5 \mu \mathrm{m}$ are achieved, corresponding to 180-pJ pulse energy. More mid-IR power is possible by further increasing the signal pulse energy while keeping the pump pulse energy below the crystal damage threshold. The limitation of crystal damage and two-photon absorption can be mitigated by replacing the $\mathrm{Yb}$-fiber laser with other fiber lasers operating at longer wavelengths, such as Er-fiber lasers at $1550 \mathrm{~nm}$. We recently applied SESS to ultrafast Er-fiber lasers and obtained femtosecond pulses tunable from $1.3 \mu \mathrm{m}$ to $1.7 \mu \mathrm{m}$ [18]. We also showed that using shorter conventional single-mode fibers can increase the filtered pulse energy up to $130 \mathrm{~nJ}$, resulting 100-fs pulses with >1-MW peak power [19]. Such an Er-fiber laser source has the potential to enable generation of nJ-level mid-IR pulses through DFG. In combination with high repetition rate, offset-free ultrafast mid-IR sources with Watt level average power are within reach. By stabilizing the repetition rate of the source laser, mid-IR laser frequency combs with unprecedented line power can be implemented.

Funding. Helmholtz Association through Helmholtz Young Investigator Group (VH-NG-804), Helmholtz-CAS Joint Research Group (HCJRG 201), and The Hamburg Centre for Ultrafast ImagingStructure, Dynamics and Control of Matter at the Atomic Scale of the Deutsche Forschungsgemeinschaft (grant EXC 1074).

Acknowledgment. The authors acknowledge Dr. Michaël Hemmer and Dr. Liwei Song for assistance in experiments.

\section{References}

1. F. Tittel, D. Richter, and A. Fried, in Solid-State Mid-Infrared Laser Sources I. Sorokina, and K. Vodopyanov, eds. (Springer 2003).

2. A. Schliesser, N. Picque, and T. W. Hänsch, Nat. Photonics 6 (7), 440-449 (2012).

3. Y. Yao and W. H. Knox, Opt. Express 21, 26612 (2013).

4. M. Beutler, I. Rimke, E. Buettner, V. Petrov, and L. Isaenko, Opt. Lett. 39, 4353 (2014)
5. M. Beutler, I. Rimke, E. Buettner, P. Farinello, A. Agnesi, V. Badikov, D. Badikov, and V. Petrov, Opt. Express 23, 2730 (2015).

6. D. Winters, P. Schlup, and R. Bartels, Opt. Lett. 35, 2179 (2010).

7. T. W. Neely, T. A. Johnson, and S. A. Diddams, Opt. Lett. 36, 4020 (2011).

8. A. Ruehl, A. Gambetta, I. Hartl, M. E. Fermann, K. S. E. Eikema, and M. Marangoni, Opt. Lett. 37, 2232 (2012).

9. C. R. Phillips, J. Jiang, C. Mohr, C. Langrock, M. Snure, D. Bliss, M. Zhu, I. Hartl, J. S. Harris, M. E. Fermann, and M. M. Fejer, Opt. Lett. 37, 2928 (2012).

10. A. Gambetta, N. Coluccelli, M. Cassinerio, D. Gatti, P. Laporta, G. Galzerano, and M. Marangoni, Opt. Lett. 38, 1155 (2013).

11. N. Coluccelli, M. Cassinerio, A. Gambetta, P. Laporta, and G. Galzerano, Opt. Lett. 39, 1661 (2014).

12. D. Sánchez, M. Hemmer, M. Baudisch, K. Zawilski, P. Schunemann, H. Hoogland, R. Holzwarth, and J. Biegert, Opt. Lett. 39, 6883 (2014).

13. H. Pires, M. Baudisch, D. Sanchez, M. Hemmer, J. Biegert, Prog. Quant. Electron. 43, 1 (2015).

14. V. Petrov, Prog. Quant. Electron. 42, 1 (2015).

15. W. Liu, C. Li, Z. Zhang, F. X. Kärtner, and G. Q. Chang, Opt. Express 24 , 15328 (2016).

16. W. Liu, S.-H. Chia, H.-Y. Chung, F.X. Kärtner, and G. Q. Chang, Opt. Express 25, 6822 (2017).

17. G. Cerullo and S. De Silvestri, Rev. Sci. Instrum. 74, 1 (2003).

18. H.-Y. Chung, W. Liu, Q. Cao, F. X. Kärtner, and G. Q. Chang, Opt. Express 25(14), 15760 (2017).

19. H.-Y. Chung, W. Liu, Q. Cao, L. Song, F. X. Kärtner and G. Q. Chang, Opt. Express 26, 3684 (2018). 


\section{Full References List}

1. F. Tittel, D. Richter, and A. Fried, "Mid-infrared laser applications in spectroscopy solid-state mid-infrared laser sources," in Solid-State MidInfrared Laser Sources I. Sorokina, and K. Vodopyanov, eds. (Springer 2003).

2. A. Schliesser, N. Picque, and T. W. Hansch, Nat. Photonics 6 (7), 440 (2012).

3. Y. Yao and W. H. Knox, Opt. Express 21, 26612 (2013).

4. M. Beutler, I. Rimke, E. Buettner, V. Petrov, and L. Isaenko, "Differencefrequency generation of $\mathrm{fs}$ and $p s$ mid-IR pulses in LilnSe $\mathrm{e}_{2}$ based on $\mathrm{Yb}$ fiber laser pump sources," Opt. Lett. 39, 4353 (2014).

5. M. Beutler, I. Rimke, E. Buettner, P. Farinello, A. Agnesi, V. Badikov, D. Badikov, and V. Petrov, "Difference-frequency generation of ultrashort pulses in the mid-IR using $\mathrm{Yb}$-fiber systems and $\mathrm{AgGaSe}_{2}$," Opt. Express 23, 2730 (2015).

6. David G. Winters, Philip Schlup, and Randy A. Bartels, "Subpicosecond fiber-based soliton-tuned mid-infrared source in the 9.7-14.9 $\mu \mathrm{m}$ wavelength region," Opt. Lett. 35, 2179-2181 (2010).

7. Tyler W. Neely, Todd A. Johnson, and Scott A. Diddams, "High-power broadband laser source tunable from $3.0 \mu \mathrm{m}$ to $4.4 \mu \mathrm{mbased}$ on a femtosecond Yb:fiber oscillator," Opt. Lett. 36, 4020-4022 (2011).

8. Axel Ruehl, Alessio Gambetta, Ingmar Hartl, Martin E. Fermann, Kjeld S. E. Eikema, and Marco Marangoni, "Widely-tunable mid-infrared frequency comb source based on difference frequency generation," Opt. Lett. 37, 2232-2234 (2012).

9. C. R. Phillips, J. Jiang, C. Mohr, C. Langrock, M. Snure, D. Bliss, M. Zhu, I. Hartl, J. S. Harris, M. E. Fermann, and M. M. Fejer, "Widely tunable midinfrared difference frequency generation in orientation-patterned GaAs pumped with a femtosecond Tm- fiber system," Opt. Lett. 37, 2928 (2012).

10. Alessio Gambetta, Nicola Coluccelli, Marco Cassinerio, Davide Gatti, Paolo Laporta, Gianluca Galzerano, and Marco Marangoni, "Milliwattlevel frequency combs in the 8-14 $\mu \mathrm{m}$ range via difference frequency generation from an Er:fiber oscillator," Opt. Lett. 38, 1155-1157 (2013).

11. Nicola Coluccelli, Marco Cassinerio, Alessio Gambetta, Paolo Laporta, and Gianluca Galzerano, "High-power frequency comb in the range of 2-2.15 $\mu \mathrm{m}$ based on a holmium fiber amplifier seeded by wavelength-shifted Raman solitons from an erbium-fiber laser," Opt. Lett. 39, 1661-1664 (2014).

12. D. Sánchez, M. Hemmer, M. Baudisch, K. Zawilski, P. Schunemann, H. Hoogland, R. Holzwarth, and J. Biegert, "Broadband mid-IR frequency comb with CdSiP2 and AgGaS2 from an Er,Tm:Ho fiber laser," Opt. Lett. 39, 6883-6886 (2014).

13. H. Pires, M. Baudisch, D. Sanchez, M. Hemmer, J. Biegert, "Ultrashort pulse generation in the mid-IR," Prog. Quant. Electron. 43, 1 (2015).

14. V. Petrov, "Frequency down-conversion of solid-state laser sources to the mid- infrared spectral range using non-oxide nonlinear crystals," Prog. Quant. Electron. 42, 1 (2015).

15. W. Liu, C. Li, Z. Zhang, F. X. Kärtner, and G. Q. Chang, "Self-phase modulation enabled, wavelength-tunable ultrafast fiber laser sources: an energy scalable approach," Opt. Express 24 (14), 15328-15340 (2016).

16. W. Liu, S.-H. Chia, H.-Y. Chung, X. Kärtner, and G. Q. Chang, "Energetic ultrafast fiber laser sources tunable in 1030-1215 nm for deep tissue multiphoton microscopy," Opt. Express 25 (6), 6822-6831 (2017).

17. G. Cerullo and S. De Silvestri, "Ultrafast optical parametric amplifiers," Rev. Sci. Instrum. 74, 1-18 (2003).

18. H.-Y. Chung, W. Liu, Q. Cao, F. X. Kärtner, and G. Q. Chang, "Er-fiber laser enabled, energy scalable femtosecond source tunable from 1.3 to $1.7 \mu \mathrm{m}$," Opt. Express 25(14), 15760-15771 (2017).

19. H.-Y. Chung, W. Liu, Q. Cao, L. Song, F. X. Kärtner and G. Chang, "Megawatt peak power tunable femtosecond source based on self-phase modulation enabled spectral selection," Opt. Express 26, 3684 (2018). 